\title{
The new NHS and diabetes care
}

\author{
MICHAEL A BAXTER
}

\begin{abstract}
The National Health Service (NHS) is under unprecedented pressures created by the increasing healthcare demands of a rapidly ageing population and the unsustainable cost of providing care in the traditional hospital-centred model. These pressures manifest themselves financially, but also in adverse clinical outcomes and patient experiences. Transformative changes are required, not only for system survival but, also, to create a system fit for purpose in the rapidly changing socio-political environment. The responsibility for re-engineering NHS services is placed on 211 Clinical Commissioning Groups using competitive tendering as the lever for change. The presumption is that competition will drive increased quality and reduced cost by promoting efficiency and innovation. High value has been placed on patientcentred integrated care and partnership working. The issues in diabetes care mirror the broader NHS. This review describes key features for an alternative model of care for diabetes, which takes advantage of the current priorities and initiatives in the new NHS. "Innovative" changes are rarely without problems and challenges and these "Wicked Issues" are identified and potential solutions discussed. The time is right to consider transformative changes that can improve patient care and develop, improve and protect the specialty of diabetes.
\end{abstract}

Br J Diabetes Vasc Dis 2014;14:87-94

Key words: transformational change, alternative models of care, integrated healthcare delivery, Wicked Issues, competitive tendering, commissioning levers

\section{Introduction}

Despite an annual budget in 2012/2013 of over $£ 100$ billion, 1 the NHS is facing some of the biggest challenges in its history with a particular focus on the quality of care and financial sustainability. ${ }^{2-4}$ The architects of this dilemma are listed in Table 1. It is regularly stated that the solution to these issues requires fundamental changes in how we approach and deliver healthcare. ${ }^{5}$ These "fundamental" changes in healthcare need to be

Address for correspondence: Dr Michael Baxter Runnymede Hospital, Chertsey, Surrey, KT16 OPZ, UK. Tel: +44 (0)7789918360

E-mail: mabmotorhead@aol.com

http://dx.doi.org/10.15277/bjdvd.2014.026

$\begin{array}{ll}\text { Abbreviations and acronyms } \\ \text { AHSN } & \text { Academic Health Science Network } \\ \text { AQP } & \text { any qualified provider } \\ \text { BCF } & \text { Better Care Fund } \\ \text { CCG } & \text { Clinical Commissioning Group } \\ \text { FT } & \text { Foundation Trusts } \\ \text { GP } & \text { General Practitioner } \\ \text { HCP } & \text { health care professional } \\ \text { LAT } & \text { local area team } \\ \text { NaDIA } & \text { National Inpatient Diabetes Audit } \\ \text { NHS } & \text { National Health Service } \\ \text { PPM } & \text { Primary Provider Model } \\ \text { QIPP } & \text { Quality, Innovation, Prevention, Productivity } \\ \text { QOF } & \text { Quality and Outcomes Framework }\end{array}$

Table 1 Drivers of the NHS crisis

- A rapidly ageing population, with increasing frailty and dependence on healthcare

- Increasing prevalence of long term conditions (LTC) e.g. diabetes, which are associated with increased morbidity and comorbidity

- Rapid rises in the prevalence of dementia with implications for long term care.

- Rapid rises in obesity with implications for long term health via increased rates of cancer, cardiovascular disease and diabetes

- Over dependence on a model of care that centres on acute hospital delivered care. This is increasingly viewed as highly costly and in some situations, particularly in the elderly and LTC, may be inappropriate.

- Medical advances which include investigations, procedures and treatment which, although designed to improve outcome, have high acquisition costs and often, at least at the point of initiation, unproven, and unquantified impact on outcome and costs.

- Increased demand for care often based on a requirement for general and holistic care.

- An increased emphasis on the technical and governance aspects of nursing, reducing direct patient care

- The increased specialisation of hospital doctors, leading to a lack of a general and holistic view of patients' problems, thereby increasing the doctor-to-doctor referral rate which impacts on the continuity of care and patient-centred care.

- A reduction in the continuity and expertise of care in primary care leading to a crisis management model of care which increases acute referrals to hospital.

made in the face of increasing austerity. The challenge is to maintain or improve quality while reducing cost. 6,7

The Department of Health has suggested that $£ 20$ million needs to be removed from the recurrent costs of the NHS over the next 3 years. ${ }^{7}$ In addition, the NHS needs to make 3-5\% cost improvements annually which, over the next 5 years, amounts to a further reduction of $15-25 \%$ of current budgets. ${ }^{8}$ Furthermore, acute providers face a further $2-5 \%$ reduction in income 
as the BCF ring fences $f 3.6$ billion nationally to drive the development of integrated health and social care services. ${ }^{9}$

This process of reducing baseline funding has been termed the QIPP initiative. ${ }^{7}$ It is argued that savings can be generated by increasing Productivity (P), the Prevention of spend (P) (avoiding waste) and the rapid harnessing and assimilation of Innovation (I). Quality (Q) defines the NHS imperative that the increased focus on financial sustainability cannot be at the expense of any element of the quality paradigm (outcomes, safety and experience). It is argued that through innovation and efficiently delivered care, there will be improved outcome and, ultimately, reduced costs.

The creation of a competitive system fuelled by choice has been a long term NHS strategy. It was argued that the creation of FTs (sovereign, clinically led membership organisations) which were empowered to innovate and generate financial surplus would drive system changes, service improvements and cost effective, high quality care. ${ }^{10}$ However, the isolated empowerment of acute providers has not created the collaborative environment, now recognised as essential to generate whole system improvements. Indeed, the creation of sovereign organisations within a competitive landscape has increased the potential for protectionism and vested self-interest. This has been fuelled by fixed-tariff reimbursement, clinical up-coding and by the perverse incentives generated by payment by results. ${ }^{11}$ The creation of FTs has even failed to deliver the expected improvement of quality of care in the acute providers. Indeed, the pressure of delivering financial targets has been stated as the reason that some FTs have been the poorest performing Trusts in the country. ${ }^{12}$

It is now widely recognised that changing the healthcare dynamic requires transformational system re-engineering. The rallying call is for dramatic and truly innovative interventions to change the configuration, function and culture of the whole NHS. ${ }^{5,13}$ The focus is now on integrated care (community, primary, secondary and social care), ${ }^{14}$ honouring "Parity of Esteem" (mental health on an equal standing to physical health) ${ }^{15}$ and a comprehensive, patient centred care system which is financially sustainable and committed to improving the NHS key clinical outcomes (Table 2). ${ }^{16,17}$

Since 2013, the power and responsibility to effect system change has moved from the Department of Health, acting via 12 strategic health authorities and 60 Primary Care Trusts, to 211 CCGs. ${ }^{18}$ CCGs are locality based, membership organisations, led by local GPs and are responsible to the local population for commissioning, and overseeing care delivered from secondary and community care and independent providers. Nationally, CCGs have budgets totalling $\mathrm{f} 89$ billion and have been tasked with changing the shape of healthcare by subjecting existing contracts to competitive tender, open to a range of providers; the AQP initiative.

$A Q P$ is designed to encourage providers to develop original and collaborative solutions to ensure cost effective, high quality care characterised by high levels of patient involvement designed to improve patient outcomes and experience. ${ }^{19,20}$

The objective is that the NHS will be re-engineered by pa-
Table 2 NHS outcome domains and key objectives

\begin{tabular}{|c|c|}
\hline Domains & Objectives \\
\hline Reduce avoidable mortality & $\begin{array}{l}\text { Reduce avoidable mortality for people } \\
<75 y \text { rs old }\end{array}$ \\
\hline $\begin{array}{l}\text { Improve care in long term } \\
\text { conditions }\end{array}$ & Reduce avoidable admissions \\
\hline $\begin{array}{l}\text { Improve outcome from } \\
\text { acute illness }\end{array}$ & $\begin{array}{l}\text { Reduce avoidable length of stay } \\
\text { Increase independence on discharge }\end{array}$ \\
\hline Improve patient experience & $\begin{array}{l}\text { Improve experience of inpatients } \\
\text { Improve experience of outpatients }\end{array}$ \\
\hline Improve safety & Reduce avoidable inpatient deaths \\
\hline
\end{tabular}

tients and primary care physicians, who have a working experience of the whole healthcare system. ${ }^{21}$ It should be noted that, although CCGs hold the budget for provider services, NHS England currently retains the budgets and overseeing role of primary care ( $\mathrm{f} 18$ billion) and specialist services ( $\mathrm{f} 18$ billion), as well as public health. ${ }^{18}$ This function is discharged for NHS England via regional LATS. The LATS also provide some guidance, support and oversight to the CCGs within their region. The potential to influence primary care services is a major challenge to, and a potential conflict of, interests for CCGs.

There is a recent move for NHS England to divest some of the responsibilities for primary care to CCGs in an initiative called "co-commissioning". Although this has been broadly welcomed, the exact details of what this will look like, and how it will address the possible issues surrounding conflicts of interest have yet to be fully resolved. 22,23

Currently the major financial issue, and the focus of attention for many CCGs is the overspend on emergency care in the acute providers. ${ }^{24}$

\section{Partnership and integration}

The creation of 15 AHSNs across the country was an NHS initiative that recognised that true innovation requires inputs from diverse partnership, with a mechanism for the rapid and widespread implementation of ideas into active clinical practice. AHSN seeks to bring together University, NHS and commercial interests in order to accelerate the introduction of useful interventions in healthcare. ${ }^{25}$

The Government have also challenged the NHS to exploit potential, more difficult partnerships, particularly with big pharma or independent providers. One such partnership is the i3-Diabetes programme which is an alliance between an academic health science network and a pharma company, based on partnership working. These partnerships have been historically difficult, and although the pursuit of such partnerships is seen as potentially offering major benefits, the process and mechanism for such discussions, outside the AHSN, is ill defined, and little real progress has been made. 26,27

The commitment to create joint working, particularly between social and health care, has been given momentum by the creation of the BCF. ${ }^{9}$ 
The BCF draws monies from health and social care to create a national fund of $\mathrm{f3.8}$ billion with explicit objectives to:

- Reduce unscheduled admission rates by $15 \%$

- Increase scheduled care productivity by $20 \%$

- Improve outcomes for existing activity

by:

- Improved coordinated care

- Reduced reliance on institutional care

- Focus on prevention and support in the community

- True 7 day working

$\mathrm{f1.1}$ billion of this fund will be held back and only released when CCGs can demonstrate that initiatives are achieving their objectives. ${ }^{28}$

The explicit objective of this strategy is to reduce the influence of the acute providers in favour of a community based service formed by the willing partnership of existing providers, or the redesign of models of care with new providers. ${ }^{29} \mathrm{BCF}$ initiatives will be sanctioned by local Health and Wellbeing boards and are an indicator of the growing importance of local government, and the public, in agreeing the health and social care agenda. ${ }^{30}$

\section{Diabetes care and the new NHS}

It can be argued that diabetes care is an allegory for the NHS. The provision of diabetes care in the UK has been subject to critical review.

In 2012/2013 the National Audit report suggested that there were 24,000 avoidable deaths in patients with diabetes, and $84 \%$ of all patients with diabetes were at risk of developing complications due to suboptimal glycaemic control (against the national $\mathrm{HbA}_{1 \mathrm{C}}$ target of $6.5 \%$ ). ${ }^{31}$ All of the national targets set out in the Diabetes National Service Framework 10 year plan had been missed. The rates of retinopathy, renal disease and amputation, rather than going down as per plan, have increased by $118 \%, 56 \%, 26 \%$ respectively. ${ }^{32}$ There is also a fivefold variation in clinical outcomes across the country, a position described by Diabetes UK as a postcode lottery of care. ${ }^{33}$

It has been argued that diabetes care is fragmented across healthcare boundaries, with no clear lines of responsibility for the patient across a care pathway which was confusing and ill defined. ${ }^{34}$ These failings may offer an explanation for the disappointing clinical outcomes in diabetes care. Despite the UK $\mathrm{HbA}_{1} \mathrm{C}$ target of $6.5-7.5 \%$, statistics suggest that over $60 \%$ of patients with diabetes have an $\mathrm{HbA}_{1 \mathrm{C}}$ of $>8 \%$, while $20 \%$ of patients have an $\mathrm{HbA}_{1 \mathrm{C}}>9 \%$ and $12 \%$ have an $\mathrm{HbA}_{1 \mathrm{C}}>10 \% .{ }^{35}$

Furthermore, despite the emphasis on the instigation of early treatment to exploit "the glycaemic window of opportunity", most patients with diabetes are on inadequate therapy for many years before their treatment is escalated. When patients with type 2 diabetes are started on insulin they have often had suboptimally controlled diabetes for more than 10 years with $\mathrm{HbA}_{1} \mathrm{C}$ $>9 \%,{ }^{36}$ and are outside the "window of opportunity". At this stage patients may not actually benefit from intensified glycaemic control. ${ }^{37}$

The "damning" conclusion of the National Audit Office was that diabetes care in the UK was provided in a fragmented process, with no one taking overall responsibility for the care pathway, a process which provided unacceptable outcomes and did not represent good value for money. ${ }^{34}$ This has to be seen as an immediate call to arms.

\section{Current costs of diabetes care}

The annual costs of providing direct diabetes clinical care is estimated at $£ 10$ billion per annum or $£ 1$ million every hour. ${ }^{31,38}$ of this budget $80 \%$ is spent on the complications of diabetes, while less than $20 \%$ is spent on the early stages of the condition, when the impact of glycaemic control on the incidence of complications may be most effective. ${ }^{39}$

It is often quoted that spend on diabetes treatments accounts for $10 \%$ of the NHS drug budget. However, this accounts for less than $7 \%$ of the total spend on diabetes which is, in fact, dominated by the cost of treating late stage complications. ${ }^{39}, 40$ Costs of diabetes care are projected to double by 2030 in line with the increasing prevalence of the condition. ${ }^{41}$

Diabetes care, like the NHS, needs a fundamental redesign. The current system of diabetes care is based on a medical model which is HCP centred, based on individual face-to-face consultations, with directed care often protocol driven. Specialist care, when accessed, is exclusively hosted in a hospital setting. The model is costly and ineffective, failing to deliver patient focused care, improved clinical outcomes or improved patient experience.

\section{Possible immediate solutions to the problems of diabetes}

To mitigate the problems of diabetes a new model of care needs to address three distinct areas:

1. An efficient and clearly defined "integrated" care pathway which is patient focused and encompasses the total patient journey, irrespective of where care is provided (home, community or hospital)

2. The effective use of therapies providing appropriate and cost effective interventions, providing patients with specific care to improve clinical outcomes and clearly demonstrating VALUE to the patient and the local health economy.

3. The prevention of diabetes by the instigation of a clear public health campaign.

The rest of this review discusses the development of an integrated care pathway, but this in no way diminishes the importance of the other objectives.

\section{Past experiences}

There have been attempts to develop and improve diabetes services in the past, ${ }^{42,43}$ most of which have been initiated by the acute provider and are incremental improvements in local systems, often driven by strong, committed local personalities. Although these provide incremental improvements and address some of the recognised issues and problems, none have been transformational enough to effect the major changes that are now required.

The lack of progress may reflect a number of influences, listed in Table 3. 
Table 3 Failure to effect radical solutions

- Restricted spheres of influence over individuals which have been insufficient to generate system change

- Historical loyalties, including the position of contracts.

- The belief that each locality requires a unique solution dictated by local needs and preferences

- The drive, particularly in FT organisation, to make a profit and sustain the Payment by Results model of care. This creates a perverse incentive to resist, and indeed block initiatives for change

- The motivation, and incentive to effect radical change in a complex, and unreceptive healthcare environment. Recognising that the "core elements" of the solution are common and shared and could be mirrored and adopted

- True understanding of the potential benefits of alternative models of care for long term conditions

It is clear that local defined solutions are unlikely to produce a definitive "generic" solution to the problems of diabetes care.

There is a requirement to think about the organisation and process requirements of an alternative, modern model of care that is fit for purpose, and can exploit the opportunities generated by the current changing healthcare landscape. These changes need to be transformative, creating a model that can be applied across the country at pace and scale.

The next section in this paper attempts to present some thoughts about how transformative change might be made. These thoughts are the first steps in considering the design of a new diabetes healthcare model and are intended to fuel a debate that can generate a detailed definitive model.

\section{Primary provider/integrated healthcare models and diabetes}

The recent circulation of a Diabetes Commissioning Framework (unpublished: NHS England) is an important step in trying to provide a national template for diabetes care.

The document is well written but describes a model of best practice which sees distinct functions based on historical structures (primary, community and secondary care). This seems to be a more definitive re-statement of how diabetes services should be delivered in the old NHS landscape. A more transformational model needs to be firmly based on the delivery of integrated, holistic care where the responsibility and governance for the whole clinical pathway is much more explicitly defined.

The primary provider/integrated healthcare models are more radical ways of thinking about clinical service provision. These models, such as Kaiser Permanente in the USA and managed care networks in the UK, look to create a single "organisation" entity that is responsible for the delivery of care across a whole clinical pathway. ${ }^{44}$ The classical boundaries between primary, community, secondary and tertiary care are lost in favour of an organisation which ideally would encompass all these aspects of care delivered by clinical staff who are employed by, and have a vested interest in, the organisation. The organisation is created with clear operational objectives, clear lines of responsibility, an overriding process of governance and accountability and a clear culture and behavioural value set. 45,46

The Derby model is closely aligned to a PPM. It has created a "not for profit" organisation which delivers community diabetes care. It has "partners" in both secondary and primary care, and has a clear process for patient flow, performance management and clinical governance. ${ }^{47}$ The model does not however control the whole of the clinical pathway. Some aspects of primary care and pre-specified aspects of secondary care are exempt from the model. Secondary, in-patient care is also outside the service specification. Although the service does control elements of the diabetes budget (community budget), it does not control the secondary care diabetes budget or aspects of the primary care spend. Furthermore there is no single, attributed responsibility for the whole of the clinical pathway (King, personal communication). Although the Derby service has been very successful and received many commendations for its innovative approach, it has recently been forced into a full competitive retendering process, which is currently in progress, with its CGG.

Others have also been exploring the development of an integrated healthcare/prime provider model, Royal Wolverhampton NHS Trust (Singh, personal communication) and West Hampshire community diabetes service (Fayer, personal communication) and thinking about the key attributes of a fully integrated diabetes healthcare model and the problems with operating a highly complex organisation using a dispersed leadership structure. ${ }^{48}$

There is general agreement that the key fundamental points include: seamless integration with a single patient pathway, an overreaching governance and performance monitoring process, clear allocated accountability and responsibility for the whole "organisation", and full financial control, to allow the appropriate allocation of resources. There must be deliverables (objectives) and performance targets. The importance of creating a value based organisation with strong leadership and a defined culture and behaviours is also recognised as being fundamentally important. ${ }^{48}$

Patients need to be closely involved in the design and monitoring of the service, with clear facilities for consultation about change and process for review on ongoing performance is essential. The possibility of creating a membership organisation such as John Lewis or FTs where staff and consumers are encouraged to be part of the organisation need to be considered. $48,49,50$ The idealised key fundamentals of such an organisation are summarised in Table 4.

This organisation may be structurally real (a company) or may be a "virtual" entity (network). It may be formed by a number of different groups, summarised in Table 5 . This is not an exhaustive list but is recorded to illustrate the possibilities and to stress that in a commercial, competitive situation there will be interests both from established commercial organisations and entrepreneurial individuals in bidding to provide diabetes services.

The position of GPs as acute providers for diabetes has been confused by the potential "conflict of interest" generated by the relationship of CCGs (composed of GPs) being the commissioners of services provided by GPs. However, the move of NHS England to create a platform for the CCGs to be involved in the commission (and performance management) of primary care 
Table 4 Operational objectives of a diabetes organisation

1. To have and control its own budget in order to make appropriate investments and disinvestments, and change funding priorities.

2. To have an overall guiding and inspirational leader, with a clearly articulated and compelling service vision and well defined operational priorities. This individual takes responsibility for the management of the whole pathway and delivery of the objectives.

3. To have created common, agreed pathways and protocols of care, shared formulary and quality outcomes. The whole system would be covered by a common clinical governance process

4. To have clearly defined SMART objectives and KPIs which are routinely audited and subject to performance management reviews.

5. All staff sharing common values, culture and behaviours.

Committed to delivering a high quality, patient focused service that values compassion and personal responsibility. Where possible, all contract of employment, and job plans, would reside with the "organisation".

6. A strategy for engaging with and managing care delivered in a community/primary care setting.

7. An SLA to deliver specialist acute care and in-patient specialist care to all patients with diabetes who need to be seen in the acute trust. This would include antenatal, and where appropriate prenatal, care to women with pregnancies complicated by diabetes.

8. An SLA with specialist services (e.g. vascular and renal services) to both provide, and receive, timely support and intervention for all patients seen in their services

9. To provide appropriate and timely patient centred and cost effective interventions which have demonstrable added value and improve patient outcomes and experience.

10. To involve patients in the design of the service and to capture their voice as a force to effect change and improve service delivery

11. The development and use of a common IT system that creates a common patient record that is accessible by all agencies involved in the delivery of care.

$\mathrm{IT}=$ information technology $\mathrm{KPI}=$ key performance indicators

SLA = service level agreement

Table 5 Potential partners in a diabetes organisation

- An existing independent healthcare provider

- A new venture company in diabetes (e.g. GPs and Consultants)

- A satellite company of current provider (s) (e.g. acute trust (s), acute trusts with community providers or other commercial entity)

- A virtual trading company housed within, but separate from, an existing provider (e.g. acute trust)

- Commercial partnership of NHS/academic/commercial interests

- "Not for profit" organisation.

- A GP provider organisation

(co-commissioning) may be a signal that there is an appetite for creating a process that circumvents "conflicts of interests" and allows the closer management, and potential further involvement, of primary care as service providers.

At face value there seem to be exciting possibilities for alternative models of care for diabetes. However, one of the reasons they do not already exist is that there are very real potential problems with effecting this, or any, change. These problems must be acknowledged and our challenge is to confront and overcome these issues before true transformational change can take place. These are difficult and fundamental issues which have (singularly or collectively) the potential to prevent true progress. These issues have been termed the "Wicked Problems". ${ }^{51}$

\section{Wicked Problems}

\section{Perception of the service}

The delivery of care by a specialist organisation, potentially seen as distinct from the NHS, may be perceived as a form of privatisation. ${ }^{52}$

New market entrants would need to rapidly establish credibility and strong relationships with patients and HCP. The vision and objectives of the organisation would need to be articulated and the culture and behaviours of the organisation would need to be clearly demonstrated to service users. The engagement and stories of the patients using the service may provide very powerful advocacy. It would be essential to provide clear messages and demonstrate an effective communication strategy. ${ }^{53}$

\section{Budget and funding}

Identification and attribution of the appropriate diabetes budget may be difficult, but it is essential to fully empower the organisation and allow the investment, and disinvestment in the service as required.

The cost of secondary care outpatients would be relatively straightforward to estimate. However, the monies spent on inpatient episodes may be more difficult to calculate directly as they are not only reflective of patients admitted with direct diabetes problems but also the effect of diabetes on patients admitted with complex comorbidity which impacts on the length of stay and mortality. The recent national audit of inpatient care (NaDIA, 2014) highlights the poor provision of inpatient care and points to the improvement in standards that is required. These data may prompt an interest in the real cost of providing effective inpatient care to the standard expected in a modern hospital. 54

The identification of monies spent in primary and community care may be complex to quantify. ${ }^{54}$ There may be a requirement to produce an indicative estimate of these sums with an agreed system of review and budget correction. ${ }^{55}$

Despite the difficulties, it is fundamentally important that the budget becomes real. Financial power within the system is essential to effect changes in service priority and structure. ${ }^{56}$ There is a requirement to reduce the overall costs of diabetes care and generate a credible business plan which includes a cost improvement programme. ${ }^{57,58,59}$ Areas of potential cost containment are listed in Table 6.

\section{Relationship with the acute provider and diabetes specialists}

The contracts of most diabetes specialists are held by the acute provider. A majority of diabetologists would consider themselves to be hospital based doctors, although the numbers of community diabetologist posts are increasing and proving to be extremely popular.60 Despite their specialisation, most diabetologists split their working time between diabetes, endocrinology, acute general medicine and inpatient care.

Most estimates of the diabetes medical workforce, based simply on head count, overestimate the diabetes workforce by 
Table 6 Areas for cost containment of the diabetes service

- The creation of new lower tariffs

- Bundling of costs as a package of care, such as a single cost for a year of treatment

- Cost containment by greater productivity and more effective intervention focused on self help

- More effective use of staff, with containment of staff costs

- Reduced infrastructural "on costs" by use of less high tech and more accessible sites for service delivery e.g. GP surgeries, community premises

- Drug cost containment by effective use (section 3.2.1) and innovative business arrangements with Pharma including bundle costing, risk share and outcome delivery. These arrangements could include formal partnerships in the delivery company.

- Reduced acute admissions and long term, high cost diabetic complications

up to $60 \% .{ }^{61,62}$ The service transformation suggested here provides an opportunity to increase the productivity in diabetes care, simply by allowing specialists to focus on their area of expertise.

Although most problems in diabetes can be addressed in a community setting, and it is estimated that $80 \%$ of care is currently provided in a community setting, some issues do require hospital attendance or admission. ${ }^{43,63,64}$

Up to $25 \%$ of hospital inpatients have diabetes as a co-morbidity to their presenting problem. Diabetes can impact on the length of stay, risk of re-admission and overall mortality of inpatients. There is strong evidence that outcomes are improved when diabetes specialists are involved. It is essential that there is a continued relationship between diabetes specialists and the acute provider, but the nature of this relationship needs re-negotiation. ${ }^{65,66}$

Ideally, within a locality, diabetologists responsible for diabetes care should also provide an acute and inpatient service. A focus on specialist inpatient care should improve the quality of inpatient care and give diabetes specialists more time focused on patients with diabetes. The focus on diabetes as a clear speciality with acute, community and service management roles may also revitalise interest in diabetes as a legitimate career choice, and address the recent decline in trainee doctors expressing an intention to become diabetes specialists. ${ }^{60,61}$

\section{Primary care and the diabetes organisation}

Primary care delivers $80 \%$ of diabetes care. ${ }^{62}$ QOF and Local Enhanced Service payments have been used to incentivise the delivery of diabetes care in primary care. ${ }^{67,68}$ Although this has produced an improvement in diabetes care nationally, the standard of diabetes care is highly variable and the improvement in the management of diabetes in the poorest performing practices is very difficult. ${ }^{33}$

Primary care practices are independent contractors to the $\mathrm{NHS}$ and are currently regulated by NHS England via the LATS. ${ }^{18}$

This independent status, the relationship with a distant commissioner (NHS England), and the lack of a direct influence of CCGs provides one of the most difficult issues for the delivery of integrated diabetes care.

There may be some encouragement that help is at hand to
Table 7 Potential configurations for diabetes care in primary care

- The "diabetes organisation" holds an indicative budget for primary care including the LES and QOF payments. The organisation then manages primary care delivery directly against an SLA which balances payment against performance and quality. This would be a unique example of an external organisation directly managing primary care function, and may be made possible by co-commissioning.

- $\quad$ Practices form an operational cluster, where a hub practice with a specialist interest in diabetes supports (and helps manage) spoke practices which provide more basic diabetes services. The hub will take a line responsibility for the spoke practices and have a direct relationship with the diabetes organisation (hub and spoke model)

- $\quad$ Practices form operational clusters (Federation). Within the clusters one practice contracts directly to provide diabetes care for all the patients with diabetes within the cluster. Other individual practices will provide speciality care e.g. asthma, dementia, heart failure for patients within the cluster. The total cluster income from these services would then be divided between the practices. (Federate model).

- $\quad$ Practices are offered the ability to opt out of providing diabetes care, and patients are then seen in practices that are increasingly specialised in diabetes care and have a direct relationship with 'The diabetes care provider' (Rationalisation model).

- GPs form an independent venture company to tender for the provision of diabetes care as an independent provider.

$\mathrm{GP}=$ general practitioner; LES = local enhanced service $\mathrm{SLA}=$ service level agreement, $\mathrm{QOF}=$ quality outcomes framework

address this issue. NHS England have recently opened a dialogue on the possible sharing of primary care commissioning with CCGs (co-commissioning) and this is widely seen as an opportunity to redesign the model of primary care and potentially facilitate the developments described in this paper.22,23,68

Several potential options for the re- configuration of diabetes care in primary care are listed in Table 7. These present exciting possibilities that may facilitate the implementation of a transformational service model in which specific primary care practices (and GPs) hold a direct contract with, and are part of, the PPM/integrated healthcare organisation.

\section{Conclusion}

There is a clear and powerful imperative to change the model of healthcare provision. This is strongly reflected in the speciality of diabetes where the requirement to change is well understood. Service redesign is one of a number of priorities, but must be seen as an essential element for immediate change.

The requirement is for fundamental, radical redesign rather than further incremental improvement.

Previous attempts to improve care delivery, which is seen as fragmented and inadequate, have been hindered by a number of issues. The concept of a PPM/integrated healthcare model provides possible solutions to all the issues identified in the current model. These potential models offer ideas that could shape a truly transformational change while exploiting the recent changes and uncertainties of the present NHS landscape.

There are clearly many problems that could hinder and derail implementation of any model based on these ideas. These are 


\section{Key messages}

- The NHS and diabetes care require a transformational change to improve outcomes, reduce costs and improve patient engagement and experience

- Organisational structures are needed for integration of care, with the whole of the patient pathway under a single performance and governance process with clear lines of responsibility and accountability

- "Wicked" issues will need to be recognised and addressed to allow true transformation and the provision of a generic template for a service design for rapid implementation across the NHS

the wicked issues which have historically not been addressed. All these problems can be foreseen and are resolvable, with some, arguably the most difficult, actually being addressed by recent, potentially exciting, changes in the way primary care will be commissioned, managed and operated in the future.

The question that remains is whether, having recognised and described the problem, and identified the elements of a transformational model that would address these issues, we have the individual and collective will to move the theory, with all its potential, into an operational reality.

\section{Conflict of interest None. Funding sources None.}

\section{References}

1. Harrison A. Spending on health care: how much is enough? King's Fund; 2006.

2. Appleby R, Crawford R, Emmerson C. How cold will it be? Prospects for NHS funding: 2011-2017; 2009.

3. Francis R. Independent Inquiry into care provided by Mid Staffordshire NHS Foundation Trust January 2005-March 2009 (Vol. 375). London: The Stationery Office; 2010.

4. Baxter MA. Does Francis ignore the health services "4 Horsemen of the Apocalypse": What does this mean for the modern NHS? Br J Diabetes \& Vasc Dis 2012;13:96-102 http://dx.doi.org/10.1177/1474651413486161

5. Black N. Can England's NHS Survive? N Engl J Med 2013;369:1-3. http://dx.doi.org/10.1056/NEJMp1305771

6. Ferlie EB, Shortell SM. Improving the quality of health care in the United Kingdom and the United States: a framework for change. Milbank $Q$ 2001;79:281-315. http://dx.doi.org/10.1111/1468-0009.00206

7. Shorney RH, Ousey K. Tissue viability: the QIPP challenge. Clin Serv J 2011;26-29. ISSN 1478-5641

8. Appleby J, Galea A, Murray R. The NHS productivity challenge; 2014.

9. Iacobucci G. Financial crisis is inevitable in the NHS by 2015-16, Kings Fund says. BMJ 2014;348:g3048. http://dx.doi.org/10.1136/bmj.g3048

10. Torgerson DJ. Randomise foundation trusts. BMJ 2003;327:291. http://dx.doi.org/10.1136/bmj.327.7409.291-a

11. Black N. Time for a new approach to assessing the quality of hospitals in England. BMJ 2013;347:f4421. http://dx.doi.org/10.1136/bmj.f4421

12. Francis R. Report of the Mid Staffordshire NHS foundation trust public inquiry: executive summary (Vol. 947). London: The Stationery Office; 2013.

13. Dias DG, Mendes C, da Silva MM. A Method for Reengineering Healthcare Using Enterprise Ontology and Lean. In Knowledge Discovery, Knowledge Engineering and Knowledge Management. Springer, Berlin and Heidelberg; 2013:243-59

http://dx.doi.org/10.1007/978-3-642-54105-6_16

14. Greaves F, Pappas Y, Bardsley M, et al. Evaluation of complex integrated care programmes: the approach in North West London. Int J Integr Care 2013;13:e006.

15. Bailey S, Smith G. Why 'parity of esteem' for mental health is every hospital doctor's concern. Br J Hosp Med 2014;75:277-80. http://dx.doi.org/10.12968/hmed.2014.75.5.277

16. Kontopantelis E, Springate D, Reeves D, et al. Withdrawing performance indicators: retrospective analysis of general practice performance under UK Quality and Outcomes Framework. BMJ 2014;348:g330. http://dx.doi.org/10.1136/bmj.g330

17. Eaton $S$, Oliver $L$, Roberts, $S$. House of care approach for patients with long term conditions works at the local level. BMJ 2013;347:f7359. http://dx.doi.org/10.1136/bmj.f7359

18. Jarman $H$. The new NHS structure is unstable. Health Serv J 2014;124;16-17.

19. Jones L, Mays N. Early experiences of any qualified provider. Br J Healthc Manage 2013:19:217-24. http://dx.doi.org/ 10.12968/bjhc.2013.19.5.217

20. Peate I, Lowbridge KL, Hayes L, et al. The NHS is up for tender. Br J Nurs 2013:22:447. http://dx.doi.org/10.12968/ bjon.2013.22.8.447

21. Greer SL, Jarman H, Azorsky A. A reorganisation you can see from space: The architecture of power in the new NHS; 2014.

22. Hudson B. Public and Patient Engagement in Commissioning in the English NHS: An idea whose time has come? Public Management Review (ahead of print); 1-16.

23. Checkland K, Coleman A, McDermott I, et al. Primary care-led commissioning: applying lessons from the past to the early development of clinical commissioning groups in England. Br J Gen Pract 2013;63 e611-e619. http://dx.doi.org/10.3399/bjgp13X671597

24. Iacobucci G. "Overly prescriptive" targets pose threat to clinical commissioning groups. BMJ 2013;347:f6090. http://dx. doi.org/10.1136/bmj.f6090

25. Ovseiko PV, Heitmueller A, Allen $P$, et al. Improving accountability through alignment: the role of academic health science centres and networks in England. BMC Health Serv Res 2014;14:24. http://dx.doi.org/10.1186/1472-6963-14-24

26. Yamada T. Partnership between NHS and pharmaceutical sector is needed. BMJ 2001;322:930, 1

27. McKee S. Novo and King's Health Partners Launch Diabetes Scheme. Pharma Times Online 2014.

28. Shortell S, Addicott R, Walsh N, et al. Accountable care organisations in the United States and England; 2014.

29. Haas L, Maryniuk M, Beck J, et al. National standards for diabetes selfmanagement education and support. Diabetes Care 2013;36(Suppl1), S100-S108. http://dx.doi.org/10.2337/dc13-S100

30. Coleman A, Checkland K, Segar K, et al. Joining it up? Health and Wellbeing Boards in English Local Governance: Evidence from Clinical Commissioning Groups and Shadow Health and Wellbeing Boards. Local Government Studies 2014; (ahead of print), 1-21.

31. Square T, Lane B. Diabetic patients developing preventable life-threatening complications in hospital; 2013. www. HSCIC.gov.uk/article/2966

32. Hull S, Chowdhury TA, Mathur R, et al. Improving outcomes for patients with type 2 diabetes using general practice networks: a quality improvement project in East London. BMJ Qual Saf 2014;23:171-6. http://dx.doi.org/10.1136/bmjqs-2013-002008

33. Diabetes UK: State of the nation 2012 report. http://www diabetes.org.uk/Documents/Reports/State-of-the-Nation-2012.pdf.

34. National Audit Office. HC 683 Session 2012-13. http://www nao.org.uk/wp-content/uploads/2013/03/Early-Action-exec-summ.pdf.

35. Esposito K, Maiorino MI, Bellastella G, et al. Comment on Khunti et al. Clinical inertia in people with type 2 diabetes: a retrospective cohort study of more than 80,000 people. Diabetes Care 2013:36:3411-7. Diabetes Care 2014:37:e112-e113. http://dx. doi.org/10.2337/dc13-2511

36. Tashkin DP, Celli B, Senn S. UKPDS and the legacy effect. JAMA 1994; 272:1497-505.

37. Kostev K, Rathmann W. Changes in time to insulin initiation in type 2 diabetes patients: a retrospective database analysis in Germany and UK (2005-2010). Prim Care Diabetes 2013;3:229-33. http://dx.doi.org/10.1016/j.pcd.2013.03.003

38. Abou-Saleh A, Haq M, Barnes D. Inpatient management of diabetes in 
adults: safety and good practice. Br J Hosp Med 2014;75:258-63. http://dx.doi.org/10.12968/hmed.2014.75.5.258

39. Klein R. The new model NHS: performance, perceptions and expectations. Br Med Bull 2007;81:39-50. http://dx.doi.org/ 10.1093/bmb/ldm013

40. Kanavos P, Van Den Aardweg S, Schurer, W. Diabetes expenditure, burden of disease and management in 5 EU countries. LSE Health and Social Care 2012.

41. Zhang P, Zhang X, Brown J, et al. Global healthcare expenditure on diabetes for 2010 and 2030. Diabetes Res Clin Pract 2010; 87;293-301. http://dx.doi.org/10.1016/j.diabres.2010.01.026

42. Freeman MS, Gillibrand WP, Newton V, et al. Engaging in a new culture of innovative collaboration for diabetes service redesign. Diabet Med 2012;29(S1):123.

43. Kar P, Meekin D, Cummings M, Cranston I. The Super Six model of diabetes care: Two years on. Diabetes Prim Care 2013;15(4):211-15.

44. Kanter MH, Lindsay G, Bellows J, et al. Complete care at Kaiser Permanente: Transforming chronic and preventative care. Jt Comm J Qual Patient Saf 2013;39:484-94

45. McComb J. Is current NHS leadership sufficient or deficient? Br J Healthc Manage 2013;19:342-7. http://dx.doi.org/ 10.12968/bjhc.2013.19.7.342

46. .Ben-Ner A. Preferences and organization structure: Toward behavioural economics micro-foundations of organizational analysis. J SocioEconomics 2013;46:87-96. http://dx. doi.org/10.1016/j.socec. 2013.08.003

47. Rea R. Integrating Diabetes care in Derbyshire [PowerPoint Slides]. http://www.kingsfund.org.uk/sites/files/kf/media/rustam-rea-integratingdiabetes-care-integrated-care-may13.pdf.

48. Choi S, Jang I, Park S, et al. Effects of Organizational Culture, Self-Leadership and Empowerment on Job Satisfaction and Turnover Intention in General Hospital Nurses. J Korean Acad Nursing Admin 2014;20:206214. http://dx.doi.org/10.11111/jkana.2014. 20.2.206

49. Lewis P. John Lewis and the benefits of mutualism. Guardian Professional; 2013.

50. Pollock AM, Price D. The break up of the English NHS: the new market bureaucracy needs information systems based on members and not geographic populations. Michael Q 2011;8:460-75.

51. Fitzgerald L, McGivern G, Dopson S, Bennett C. Making Wicked Problems Governable?: The Case of Managed Networks in Health Care. Oxford University Press; 2013.

52. Baker R, Willars J, McNicol S, Dixon- Woods M, McKee L. Primary care quality and safety systems in the English National Health Service: a case study of a new type of primary care provider. J Health Serv Res 2014;19:34-41. http://dx.doi.org/10.1177/ 1355819613500664

53. Coulson-Thomson C. Transforming Knowledge Management: A Quicker and Affordable Route to High Performance Organisations. Policy Publications; 2013.

54. Health and Social Care Information Centre. National Diabetes Inpatients
Audit; 2013.

55. Caballero AE, Davidson J, Elmi $A$, et al. Previously unrecognized trends in diabetes consumption clusters in medicare. Am J Managed Care 2013:19:541-8.

56. Brooks R, Mukherjee AK. Financial management: core concepts. Pearson; 2013.

57. Zhang X. The Framework of Management Control Mode. In Enterprise Management Control Systems in China. Springer, Berlin and Heidelberg; 2014: 183-94. http://dx.doi.org/10.1007/978-3-642-54715-7_11

58. Anantadjaya SP. Entrepreneurs vs. Business Plans: A study of Practicality and Usefulness. South East Asian Journal of Management 2013;1:14368.

59. Conway PH, Mostashari F, Clancy C. The future of quality measurement for improvement and accountability. JAMA 2013;309:2215-16. http://dx.doi.org/10.1001/jama.2013.4929

60. Gill G, Newell-Price J. Diabetes and endocrinology. In: Consultant Physicians Working with Patients 2013;91-98.

61. Diabetes UK, Royal College of Physicians. The Diabetes UKIRCP diabetes and endocrinology national manpower report 2012. London: Diabetes UK; 2012

62. Baxter M, Gadsby R, Griffiths U. Empowering primary care practitioners to meet the growing challenge of diabetes care in the community. $\mathrm{Br} J$ Diabetes Vasc Dis 2006:6:245-8. http://dx.doi.org/10.1177/14746514060060060101

63. Healy SJ, Black D, Harris C, et al. Inpatient diabetes education is associated with less frequent hospital readmission among patients with poor glycemic control. Diabetes Care 2013;36(10):2960-7. http://dx.doi.org/10.2337/dc13-0108

64. McMurray JJ, Gerstein HC, Holman RR, et al. Heart failure: a cardiovascular outcome in diabetes that can no longer be ignored. Lancet Diabetes Endocrinol 2014 http:dx.doi.org/10.1016/S2213-8587(14)70031-2

65. Rayman G, Kilvert A. The crisis in diabetes care in England. BMJ 2012 345:5-6. http://dx.doi.org/10.1136/bmj.e5446

66. Kontopantelis E, Reeves D, Valderas JM, et al. Recorded quality of primary care for patients with diabetes in England before and after the introduction of a financial incentive scheme: a longitudinal observational study. BMJ Qual Saf 2013:22:53-64.

http://dx.doi.org/10.1136/bmjqs-2012-001033

67. Gadsby R, Khunti K. PCDS survey of diabetes care delivery and local enhanced services for diabetes in 2009-10. Diabetes Prim Care 2013;15:(1) 14-18.

68. Hull S, Chowdhury TA, Mathur R, et al. Improving outcomes for patients with type 2 diabetes using general practice networks: a quality improvement project in East London. BMJ Qual Saf 2014;23:171-6. http://dx.doi.org/10.1136/bmjqs-2013-002008 